\title{
Uso de terapias complementares por mães em seus filhos: estudo em um hospital universitário
}

\author{
Use of complementary therapies by mothers in their children: \\ study at an university hospital
}

Luiza Borges Gentil ${ }^{1}$

Ana Carolina Couto Robles ${ }^{2}$

Suely Grosseman ${ }^{2}$

\footnotetext{
1 Programa de Saúde da Família, Prefeitura M unicipal deSanto Amaro dalmperatriz. Praça Governador Ivo Silveira 306, Centro. 88140-000 Santo Amaro da Imperatriz SC. luizagentil@gmail.com 2 Universidade Federal de Santa Catarina.
}

Abstract The objective of this article is to evaluate the use of complementary therapies by mothers in their children. A cross-sectional and descriptive study with 202 mothers of children that attended an U niversity H ospital wereintervi ewed. The variables analyzed were: use of complementary therapies/reasons, therapies used its purpose, effects, doctor's revelation/reasons and doctor's re action. The prevalence of complementary therapies use was of $87.6 \%$. Among the 177 mothers that used complementary therapies, many mentioned morethan onekind, asfollow: teas (72.8\%), blessings (41\%), sympathies ( $12.9 \%)$, homemade remedies/syrups (8.4\%) , prayer/promises (7.4\%), homeopathy $(4.0 \%)$, spiritual/parapsychological treatment $(4.0 \%)$, mixture of unknown substances/"garrafada" (bottled) (3\%), massage (2\%) and reiki/floral (1.5\%). The most used herbs were anise $(16.7 \%)$, chamomile $(14.8 \%)$ and mint $(10.9 \%) ; 57.6 \%$ of the mothers did not inform its use to the doctor. O ut of 499 treatments employed, there was a perception of improvement in 429 (86\%) and 2 reports of adverse effects. The prevalence of complementary therapies utilization was high, being teas the most utilized therapy and anise, chamomileand mint themost used herbs. There wasperception of improvement in most of theused therapies.

Key words Complementary therapies, Pediatrics, Education, M edical, Alternative therapies, M others
Resumo 0 objetivo deste estudo éavaliar a utilização de terapias complementares (TC) por mães em seus filhos. Trata-se de um estudo transversal, descritivo. Foram entrevistadas 202 mães de crianças assistidas em um hospital universitário. As variáveis analisadas foram: uso de terapias complementares/motivos para tal, tipos de terapias utilizadas, sua finalidade, seus efeitos, revelação ao médico/motivos para tal e reação do médico. A prevalência de uso de terapias complementares foi 87,6\%. De 177 mães que utilizavam TC, muitas mencionaram mais de um tipo, sendo: chás $(72,8 \%)$, benzimento $(41 \%)$, simpatias $(12,9 \%)$, remédios/xaropes caseiros (8,4\%), oração/promessa $(7,4 \%)$, homeopatia $(4,0 \%)$, tratamento espiritual/parapsicológico (4,0\%), mistura de substâncias desconhecidas/garrafada (3\%), massagem $(2 \%)$ e reiki/florais ( $1,5 \%)$. As ervas mais utilizadas foram erva-doce $(16,7 \%)$, camomila $(14,8 \%)$ e hortelã $(10,9 \%) ; 57,6 \%$ das mães não informaram o uso ao médico. Dos 499 tratamentos empregados, houve percepção de melhora em 429 ( $86 \%$ ) e relato de dois casos de efeitos adversos. A prevalência do uso de terapias complementares foi alta, sendo os chása terapia maisutilizada e erva-doce, camomila e hortelã as ervas mais usadas. Houve percepção de melhora na maioria dasterapiasusadas.

Pal avras-chave Terapias complementares, Pediatria, Educação médica, Terapias alternativas, $M$ ães 
Introdução

As terapias complementares (TC), também conhecidas como terapias al ternativas, integrativas ou não convencionais, constituem, segundo Barnes et al. ${ }^{1}$, um grupo de terapias e produtos que não são considerados como parte da medicina alopática e englobam diversas práticas de atenção à saúde, tais como acupuntura, homeopatia, medicina ayurvédica, naturopatia, medicina fitoterápica, terapias baseadas em dietas, quiropraxia, massagem, meditação, hipnose, yoga, orações e cura pela fé, terapia de cura por reiki, entre outras.

Muitas destas terapias são práticas antigas que foram redescobertas e não deveriam ser colocadas em oposição à medicina alopática, mas sim, em uma dimensão que as incluísse?2.

Estudos apontam frequência de uso das TC em crianças variando de $1,8 \%$ a $66 \%$, sendo encontradas prevalências mais baixas nos Estados Unidos $^{3-6}$ e no Canadá7,8, e mais altas no Reino Unido ${ }^{9}$, Dinamarca ${ }^{10}$, Austráliaa ${ }^{11}$ e Brasi ${ }^{12}$.

$\mathrm{Na}$ literatura, entre os motivos encontrados para uso deTC, estão complementação da medicina convencional, orientação médica, curiosidade em relação às TC, falta de dinheiro para aquisição da terapia convencional ${ }^{1}$, indicação de conhecidos, medo de efeitos colaterais provocados pela medicina convencional, presença de doença crônica, insatisfação com a medicina convencional ${ }^{5}$, abordagem holística e relacionamento mé dico-paciente baseado em apoio e confiança $a^{1,8,13}$.

Apesar de sua ampla utilização, nem sempre os responsáveis pelas crianças revelam ao médico o uso de TC T-12. $^{-12}$.

Levando-se em consideração esse contexto, este estudo teve como objetivo avaliar, no hospital universitário (HU) da Universidade Federal deSanta Catarina (UFSC), a utilização de terapias complementares, por mães, em seus filhos, na perspectiva de construir conhecimentos sobre 0 tema, visando contribuir para a educação e a prática médica.

\section{M etodologia}

Estudo quanti equalitativo, transversal, descritivo, com eixo temporal contemporâneo.

Os sujeitos do estudo foram 202 mães, cujos filhos estavam sendo assistidos, entre os dias 27 de julho e 25 de agosto de 2006, na emergência, enfermaria ou no ambulatório de pediatria do HU/UFSC. A seleção da amostra foi por conve- niência, com mães que estavam presentes no período matutino ou vespertino, totalizando 202 mães, estando 107 no ambulatório (53\%), 85 na emergência ( $42 \%$ ) e 10 na enfermaria (5\%).

A coleta de dados foi feita por entrevista, guiada por questionário com perguntas abertas e fechadas. As variáveis analisadas foram dados de identificação das mães (idade, profissão, número de filhos e sua idade) e dados relativos à utilização de TC (uso ou não e motivos para tal, tipos de terapias utilizadas, sua finalidade, seus efeitos, revelação ou não ao médico, motivos para tal e reação do médico).

O banco de dados foi construído no programa Epidata e a análise feita através do programa Epi 6, sendo esta descritiva e com medidas de tendência central, quando pertinentes. Para comparar variáveis contínuas, foi utilizado o teste tStudent, sendo considerado 0,05 como nível de significância para a hi pótese nula.

As questões abertas foram categorizadas e alguns termos utilizados pelas mães são apresentados como descritos por elas.

0 projeto deste estudo foi aprovado pelo Comitê de Ética em Pesquisa com Seres Humanos da UFSC (271/06).

\section{Resultados}

A média da idade das entrevistadas foi 30,8 [ desvio padrão $(D P)=7,9$ ] e o número de filhos variou de 1 a 9 , sendo a mediana 2 e a moda 2 .

Entreas 202 entrevistadas, 177 (87,6\%) referiram a utilização de TC, sen do a média da idade das mães que as utilizaram 30,9 ( $D P=8,1$ ) ea das que nunca as utilizaram 29,5 ( $D P=6,3)$, não havendo diferença estatisticamentesignificativa entre elas $(p>0,05)$.

O motivo mais citado para utilização de TC foi a influência de pessoas próximas, como pode ser observado na Tabela 1.

As mães que não fizeram uso de TC alegaram não ter sido necessário (28\%), não ter fé nestas terapias (24\%), não aceitação de chá pela criança ( $16 \%$ ), não confiar em quem não é mé dico para tratamento do filho (12\%), não ter interesse ( $8 \%$ ), estar a criança em al eitamento materno exclusivo (8\%), acreditar que as TC podem causar outro problema (4\%).

Entre as 177 mães que utilizavam TC, muitas mencionaram usar mais de um tipo, sendo os chásutilizados por $72,8 \%$ delas, o benzimento (por benzedeiras) por $41 \%$, as simpatias por $12,9 \%$, remédios/xaropes caseiros por $8,4 \%$, oração/pro- 
Tabela 1. M otivos alegados por 177 das 202 mães de pacientes pediátricos para usar terapias complementares (TC). Hospital Universitário da Universidade Federal de Santa Catarina, 2006.

\begin{tabular}{|c|c|}
\hline Motivo para uso & $\begin{array}{c}\text { Freqüência* } \\
n(\%)\end{array}$ \\
\hline Influência de pessoas próximas & $110(62,1)$ \\
\hline Ter fé na terapia & $39(22,0)$ \\
\hline Boa experiência anterior com TC & $32(18,1)$ \\
\hline Uso de substâncias naturais & $13(7,3)$ \\
\hline $\begin{array}{l}\text { Necessidade de fazer alguma coisa } \\
\text { para ajudar o filho }\end{array}$ & $10(5,6)$ \\
\hline $\begin{array}{l}\text { Terapia adjuvante ao tratamento } \\
\text { convencional }\end{array}$ & $09(5,1)$ \\
\hline Evitar terapias agressivas & $09(5,1)$ \\
\hline $\begin{array}{l}\text { Insatisfação com tratamento } \\
\text { convencional }\end{array}$ & $07(4,0)$ \\
\hline $\begin{array}{l}\text { Falta de dinheiro para compra de } \\
\text { medicamento }\end{array}$ & $06(3,4)$ \\
\hline Recomendação médica & $03(1,7)$ \\
\hline Testar a TC & $02(1,1)$ \\
\hline M orar longe de centro médico & $02(1,1)$ \\
\hline
\end{tabular}

* Os resultados totalizam mais de $100 \%$, por que foram alegados mais de um motivo, totalizando 242 entre as 177 mães

messa por 7,4\%, homeopatia por 4,0\%, tratamento espiritual/parapsicológico por $4,0 \%$, mistura desubstâncias desconhecidas (garrafada) por 3\%, massagem por $2 \%$ e reiki/florais por $1,5 \%$.

Em chás e remédios/xaropes caseiros, as ervas mais utilizadas foram erva-doce $(16,7 \%)$, camomila (14,8\%) e hortelã (10,9\%). Na Tabela 2 , constam as ervas e outras substâncias utilizadas e suas finalidades.

$\mathrm{Na}$ Tabela 3, estão descritos os efeitos dos 499 tratamentos realizados com TC. A maioria das mães relatou melhora dos sintomas em seus filhos com a terapia utilizada. Entre as duas mães que relataram piora, uma referiu agravamento dos sintomas respiratórios do quadro de asma de seu filho com o uso de chá de eucalipto euma referiu dermatite de contato por moeda colocada como simpatia, para tratar hérnia umbilical de seu filho.

A utilização de TC não foi revelada ao médico por $57,6 \%$ mães, sendo revelada por $40,7 \%$ (1,7\% não se lembravam). As orações (18,8\%), os tratamentos espírita e parapsicológico $(37,5 \%)$, as simpatias $(38,5 \%)$ e a procura por benzedeiras $(35,4 \%)$ foram pouco informados
Tabela 2. Ervas e outras substâncias utilizadas em chás e remédios/ xaropes caseiros por mães de pacientes pediátricos e sua finalidade.

\begin{tabular}{|c|c|}
\hline Tratamento de ou para & Ervas - outras substâncias \\
\hline \multicolumn{2}{|l|}{ Vias aéreas } \\
\hline "D or de garganta" & Alfavaca*, malva*, tansagem* - gengibre* \\
\hline Bronquite & 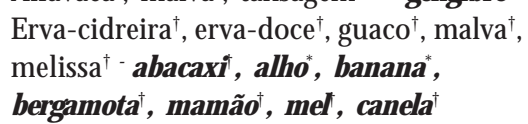 \\
\hline Gripe & 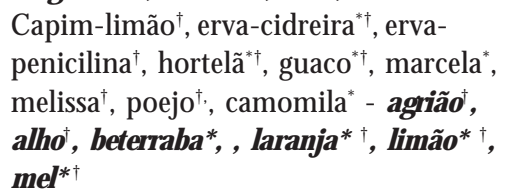 \\
\hline Tosse & $\begin{array}{l}\text { Camomila*- } \text { Abacaxi }^{\dagger} \text {, agrião }{ }^{\dagger} \text {, alho }{ }^{\dagger}, \\
\text { cenoura }{ }^{\dagger}, \text { cravo }^{\dagger} \text {, eucalipto*, Guaco*t, } \\
\text { hortelä*, marcela*, poejo*, tansagem* } \\
\text { laranja*, mel* }{ }^{*}\end{array}$ \\
\hline
\end{tabular}

Trato gastrointestinal

Vômitos

"D or de estômago"

Boldo*, camomila*, erva-doce*, hortelã* Alho ${ }^{\dagger}$, boldo*, camomila*, chá-preto*, erva-cidreira* ${ }^{\dagger}$, espinheira-santa*, hortelã ${ }^{*}+$, laranja*, louro*, marcela*, rosa branca*, sene*

Cólica

Camomila*, capim-limão*, chá-preto*, chicória†', cânfora*, erva- doce*, ervacidreira* ${ }^{\dagger}$, funcho* ${ }^{\dagger}$, hortelã*, manjerona*, marcela*, noz-moscada*, poejo* - ameixa*

Verminose Hortelã* Estimular a evacuação Diarréia Infecção urinária Dormir / acalmar

Aftas

Ameixa* goiaba*, romã

Erva-cidreira* ${ }^{\dagger}$

Calêndula*, camomila*†, capim-limão*†, erva-cidreira*†, erva- doce ${ }^{\dagger}$, ervapenicilina ${ }^{\dagger}$, hortelã ${ }^{\dagger}$ marcela $^{\dagger}$, melissa ${ }^{\dagger}$ laranja*, maracujá* ${ }^{\dagger}$, bergamota ${ }^{\dagger}$ Cravo $^{\dagger}$

Febre ou sua prevenção Erva-cidreira* ${ }^{\dagger}$ - Iaranja*

ao nascer dentes

Hipercolesterolemia M arcela*

Cobreiro

Icterícia do recém- Picão*

nascido

Adenopatia Semente de girassol*

* utilizado puro, na forma de chá; ${ }^{\dagger}$ utilizado misturado com outras substâncias (principalmentemel) e/ou ervas, na forma de chás ou xaropes.

ao médico, enquanto os xaropes $(64,7 \%)$, as massagens ( $75 \%)$, a homeopatia $(55,6 \%)$ e o reiki e os florais ( $75 \%)$, geralmente, o foram.

As razões citadas pelas mães para não revelar ao médico foram o profissional não ter pergun- 
Tabela 3. Percepção das 177 mães de pacientes pediátricos sobre o efeito dos 499 tratamentos com terapias complementares empregados por elas em seus filhos. H ospital Universitário da Universidade Federal de Santa Catarina, 2006.

\begin{tabular}{lccrr}
\hline \multicolumn{1}{c}{ Terapia utilizada } & Melhorou n (\%) & Sem efeito n (\%) & Piorou n (\%) & Total n (\%) \\
\hline Chás & $270(86,3)$ & $42(13,4)$ & $01(0,3)$ & $313(100)$ \\
Benzedeira & $82(85,4)$ & $14(14,6)$ & - & $96(100)$ \\
Simpatia & $20(76,9)$ & $05(19,2)$ & $1(3,9)$ & $26(100)$ \\
Xarope caseiro & $16(94,1)$ & $01(5,9)$ & - & $17(100)$ \\
Oração & $13(81,2)$ & $03(18,8)$ & - & $16(100)$ \\
Homeopatia & $09(100)$ & - & - & $09(100)$ \\
Parapsicologia/ tratamento espírita & $05(62,50)$ & $03(37,50)$ & - & $08(100)$ \\
Garrafada* & $06(100)$ & - & - & $06(100)$ \\
Massagem & $04(100)$ & - & - & $04(100)$ \\
Reiki/florais & $04(100)$ & - & - & $04(100)$ \\
Total de tratamentos realizados & 429 & 68 & 02 & 499 \\
\hline
\end{tabular}

*mistura com substâncias desconhecidas.

tado $(46,1 \%)$, "não ver necessidade de informálo" (23,5\%), não ter retornado ao médico após uso de TC $(16,7 \%)$ e acreditar que o médico não aprovaria a terapia ( $13,7 \%)$.

$\mathrm{N}$ a percepção das mães, a aprovação foi a reação mais frequente dos médicos ao serem informados sobre o uso de TC $(47,2 \%)$, seguida pela indiferença (38,9\%); $11,1 \%$ dos profissionais recomendaram a suspensão do uso e 2,8\%, o cuidado na utilização.

\section{Discussão}

A prevalência de mães que utilizaram TC em seus filhos neste estudo foi maior que a encontrada em estudos nacionais e internacionais. Nos Estados Unidos, Davis e Darden ${ }^{4}$ encontraram a prevalência de $1,8 \%$ de uso de TC entrea população pediátrica americana não institucionalizada; Sawni et al. ${ }^{6}$, a de $15 \%$ em pacientes abaixo de dezoito anos que procuravam a emergência de um hospital infantil de Detroit, Sawni-Sikand et al. ${ }^{5}$, a de $12 \%$ em consultas pediátricas na atenção primária em Detroit eSibinga et al. ${ }^{3}$, a de $21 \%$ em crianças que faziam consulta de puericultura ou por doença aguda na atenção primária em Washington.

No Canadá, Losier et al. ${ }^{7}$ encontraram a pre valência de 13\% na emergência de um hospital terciário em Ottawa eSpigelblatt et al. ${ }^{8}$, a de $11 \%$ hospital universitário de Montreal. No Reino Unido, Crawford et al..$^{9}$ encontraram a prevalência de $41 \%$ deuso deTC no ano anterior ao estu- do, em crianças e adolescentes que consultavam ou estavam internados no hospital universitário do país de Gales. Na Dinamarca, M adsen et al. ${ }^{10}$ encontraram prevalência de $53 \%$ nos queprocuravam qualquer setor da pediatria de um hospital de Odense. Na Austrália, Cincotta et al. ${ }^{11}$, em estudo com crianças atendidas no Hospital de M elbourne, encontraram prevalência de $51 \%$ de uso de TC no ano anterior; entretanto, no estudo em população geral de Smith e Eckert ${ }^{14} \mathrm{em}$ Adelaideesta foi de $18,4 \%$. No Brasil, Alves eSil$\mathrm{va}^{12}$, investigando em centros de saúde de São Paulo o uso de ervas em menores de cinco anos, constataram a prevalência de $66 \%$.

As diferentes prevalências dos estudos poderiam ser explicadas por fatores culturais, por diferenças no método (origem da amostra, técnica de coleta de dados, variáveis consideradas, entre outras) e por fatores relacionados aos entrevistados como o medo de revelar o uso de TC 7,8,12.

No Brasil, o uso de TC está relacionado às diferentes raízes culturais das populações que aqui residiam. Durante os três primeiros séculos da colonização, os colonizadores recorriam às formas de cura trazidas da Europa ou àquelas utilizadas por pessoas de etnias diversas, com as quais mantiveram contato. Os missionários jesuítas aproveitaram muito da medicina indígena (a primeira aqui existente), sendo a eles imputada a iniciativa de intercâmbio entre os universos da medicina. Com a chegada dos escravos africanos, a sociedade também aderiu a certas práticas de cura relacionadas à magia ${ }^{15}$. 
N este estudo, os motivos mais frequentemente alegados para utilização de TC também foram encontrados em outros estudos e foram indicação de pessoas próximas, ter féna terapia, experiência anterior positiva com $T C$, necessidade de fazer alguma coisa para ajudar o filho, auxiliar a terapia convencional, evitar terapias agressivas, insatisfação com o tratamento convencional, dificuldades financeiras para compra de medicamentos, recomendação médica, testar a terapia e morar longe de centro médico. Losier et al. ${ }^{7}$, Spigelblatt et al. ${ }^{8}$ eYatsugafu ${ }^{16}$ citam também a atenção mais personalizada e holística e o medo de efeitos colaterais dos medicamentos.

Os motivos referidos para a não utilização de TC neste trabal ho vão ao encontro daqueles encontrados em outros estudos: falta de confiança na terapia e em tratamentos que não sejam realizados por médicos, medo do desconhecido e medo de efeitos colaterais $s^{4,16}$.

No atual estudo, as TC mais utilizadas foram chás $(72,78 \%)$, procura por benzedeiras $(46,89 \%)$ e realização desimpatias ( $12,87 \%)$.

0 uso de ervas, principalmente na forma de chá $(72,78 \%)$, foi maior do que o encontrado por Kumanan et al. ${ }^{17} \mathrm{em}$ menores de dezoito anos atendidos em clínica de naturopatia no Canadá (14,9\%), por Cincotta et al. ${ }^{11}$ (10\%) e Crawford et al. ${ }^{9}(17 \%)$. Chen et al. ${ }^{18}$ encontraram $85,9 \%$ de uso de ervas entre os usuários de medicina tradicional chinesa no Taiwan.

Neste estudo, os chás de camomila, erva-cidreira e funcho foram utilizados para tratar cólicas. Segundo Rogovik e Goldman ${ }^{19}$, o chá destas três ervas associado à verbena e al caçuz é efetivo no tratamento de cólicas do lactente.

As ervas mais utilizadas para confecção de chás e remédios/xaropes casei ros foram a ervadoce, a camomila e o hortelã (10,91\%), as quais também foram as mais citadas no estudo de Alves e Silva ${ }^{12}$.

A procura por benzedeiras também foi encontrada no estudo de Yatsugafu ${ }^{16}$.

A utilização de simpatias, orações e promessas também foram citadas em outras pesquisas $5^{13,16,20}$. Queiroz e Canesqui ${ }^{20}$, em pesquisa com 889 adultos de Campinas (Brasil), constataram que $31 \%$ utilizavam simpatias para tratar a si mesmos e/ou a seusfilhos. Richardson ${ }^{13}$ encontrou $80,5 \%$ deuso de "práticas espirituais" entre 453 pacientes ambulatoriaisnos Estados Unidos, sendo $91 \%$ delas concomitantes ao tratamento alopático.

0 uso desses recursos geralmente está ligado à crença de que a causa da doença tem fundo religioso ou espiritual e, na opinião de Sloan et al. ${ }^{21}$, ai nda quenão existam evidências consistentes de associação entre religião, espiritualidadee saúde, ninguém deveria objetar-se a apoiar a fé religiosa de seu paciente.

A homeopatia foi pouco citada neste estudo $(3,96 \%)$, indo ao encontro dos dados encontrados por Kumanan et al. ${ }^{17}(3,7 \%)$, Crawford et al. ${ }^{9}(4 \%)$ e Cincotta et al. ${ }^{11}(5 \%)$.

A garrafada (mistura com substâncias desconhecidas) foi referida nos trabalhos de Lehmkuhl ${ }^{22}$, para alívio dos sintomas de quimioterapia ou cura do câncer em adultos, e de Yatsugafu ${ }^{16}$, com diversas finalidades, no tratamento de crianças.

As massagens, pouco relatadas neste e em outros estudos ${ }^{7,9-11}$, têm alta prevalência de uso (quase $100 \%$ nos neonatos) em países orientai ${ }^{23}$, com exceção de Taiwan, onde apenas 3,1\% das pessoas queutilizavam medicinatradicional chinesa referiram seu uso em estudo deChen et al. ${ }^{18}$.

Quanto aos efeitos dos tratamentos realizados com TC, enquanto neste estudo houve percepção de melhora por $86 \%$ mães, semelhante à encontrada por Clement et al. ${ }^{24} \mathrm{em}$ Trinidad, ilha localizada nas Índias O cidentais ao norte da Venezuela $(86,8 \%)$, e maior que a constatada por Spigelblatt et al. ${ }^{8}(59 \%)$ no Canadá.

Tsao e Zeltzer ${ }^{25}$ afirmam que diversas TC são promissoras no tratamento da dor em pacientes pediátricos, entre el as a acupuntura, biofeedback, ervas, massagem, homeopatia e hipnose.

A pesar dos benefícios, é fundamental que o riscos das TC sejam conhecidos pela população e por quem as indica, antes de sua utilização. Vários autores têm feito estudos e revisões sistemáticas ${ }^{26-29}$ sobre o tema. Os riscos das ervas incluem o potencial para sensibilização de numerosos remédios à base de ervas, podendo levar à dermatite de contato e, mais raramente, a sintomas clínicos mediados pela Ig-E; a possibilidade de vários preparos à base de ervas causarem toxicidadehepática, renal e/ou cardíaca, além de interagirem com outras drogas ou ervas; 0 potencial de algumas ervas para carcinogênese.

Quanto à revelação ao médico, $40,68 \%$ das mães deste estudo informaram o uso de TC em seus filhos, número próximo ao encontrado por Sibinga et al. ${ }^{3}$ nos Estados Unidos (36\%), por Cincotta et al. ${ }^{11}$ naAustrália (37\%), por Crawford et al. ${ }^{9}$ no Reino Unido (44\%) e por Jean e Cyr ${ }^{30}$ em crianças atendidas em ambulatório geral no Canadá (44\%) e mais baixo do que o encontrado por Spigelblatt et al. ${ }^{8}$ no Canadá e M adsen et al. ${ }^{10}$ na Dinamarca (50\%) e por Sawni-Sikand et al. ${ }^{5}$ nos Estados Unidos (66\%). 
Levando em consideração a alta prevalência do uso deTC por mães em seus filhos constatada neste estudo ea pouca revelação ao médicos, seria importante que os médicos questionassem mais os responsáveis por pacientes pediátricos quanto a seu uso eque tivessem maior conhecimento sobre suas possibilidades e limitações para poderem julgar a adequação ou não das TC utilizadas.

\section{Colaboradores}

LB Gentil, Robles ACC e Grosseman S participaram igualmente de todas as etapas da elaboração do artigo.

\section{Referências}

1. Barnes PM, Powell-Griner E, M cFann K, Nahin $\mathrm{RL}$. Complementary and alternative medicine use among adults: United States, 2002. Adv Data 2004; 343:1-19.

2. Queiroz MS. O itinerário rumo às medicinas alternativas: uma análise em representações sociais de profissionais da saúde. Cad Saude Publica 2000; 16(2):363-375.

3. Sibinga EMS, Ottolini MC, Duggan AK, Wilson MH. Parent-pediatrician communication about complementary and alternative medicine use for children. Clin Pediatr 2004; 43(4):367-373.

4. Davis MP, Darden PM. Use of complementary and alternative medicine by children in the United States. Arch Pediatr Adolesc M ed 2003; 157:393-396.

5. Sawni-Sikand A, Schubiner $H$, Thomas RL. U se of complementary/alternative therapies among children in primary care pediatrics. Ambul Pediatr. 2002; 2(2):99-103.

6. Sawni A, Ragothaman R, Thomas RL, Mahajan P. The use of complementary/alternative therapies among children attending an urban pediatric emergency department. Clin Pediatr 2007; 46(1):36-41.

7. Losier A, Taylor B, Fernandez CV. Use of alternative therapies by patients presenting to a pediatric emergency department. J Emerg Med 2005; 28(3):267-271.

8. Spigelblatt L, Laine-Ammara G, Pless IB, Guyver A. The use of alternative medicine by children. Pediatrics 1994; 94:811-814.

9. Crawford NW, Cincotta DR, Lim A, Powell CVE. A cross-sectional survey of complementary and alternative medicine use by children and adolescents attending the University of Wales. BM C complementary and alternative medicine [periódico na Internet] 2006 [acessado $2007 \mathrm{dez}$ 5];6(16):[cerca de 10 p.]. Disponível em: http://www.biomedcentral.com/ 1472-6882/6/16

10. Madsen $H$, Andersen $S, N$ ielsen RG, Dolmer BS, Host A, Damkier A. Use of complementary/alternative medicine among paediatric patients. Eur J Pediatr 2003; 162(5):334-341. 
11. Cincotta DR, Crawford NW, Lim A, Cranswick NE, Skull S, South M, Powell CV. Comparison of complementary and alternative medicine use: reasons and motivations between two tertiary children's hospitals. Arch Dis Child 2006; 91(2):153-158.

12. Alves AR, Silva MJP. O uso da fitoterapia no cuidado de crianças com até cinco anos em área central e periférica da cidade de São Paulo. Rev Esc Enferm USP 2003; 37(4):85-91.

13. Richardson J. What patiens expect from complementary therapy: a qualitative study. Am J Public Health 2004; 94(6):1049-1053.

14. Smith C, Eckert K. Prevalence of complementary and alternative medicine and use among children in South Australia. J Paediatr Child Health 2006;42(9):53-43.

15. Edler F, Fonseca, MRF da. Saber erudito e saber popular na medicina colonial. Cadernos da ABEM 2006; 2:8-9

16. Yatsugafu CT. U tilização de recursos terapêuticos nãoconvencionais em crianças que frequentam o $\mathrm{H}$ ospital Universitário/Universidade Federal de Santa Catarina: um estudo de caso [trabalho de conclusão de curso]. Florianópolis (SC): Universidade Federal de Santa Catarina; 2006.

17. Kumanan W, Busse JW, Gilchrist A, Vohra S, Boon $\mathrm{H}, \mathrm{Mills}$ E. Characteristics of Pediatrics and Adolescent Pacients Attending a Naturopathic College Clinic in Canada. Pediatrics 2005; 115(3):338-343.

18. Chen FP, Chen TJ, Kung YY, Chen YC, Chou LF, Chen FJ, H wang SJ. U se frequency of traditional Chinese medicine in Taiwan. BM C Health Serv Res 2007. [periódico na Internet] 2007 [acessado 2007 dez 5];7(26):[cerca de 11 p.]. Disponível em: http:/ /www.biomedcentral.com/1472-6963/7/26

19. Rogovik AL, Goldman RD. Treating infants' colic. Can Fam Physician 2005; 51(9):1209-1211.

20. Queiroz MS, Canesqui, AM . Famílias trabalhadoras e representações sobre a saúde, doença e aspectos institucionais da medicina oficial e popular. Caderno de Pesquisa do N epp UNICAM P 1989;(7):1-57.
21. Sloan RP, Bagiella E, Powell T. Religion, spirituality, and medicine. Lancet 1999; 353(9153):664-667.

22. Lehmkul RF. Recursos terapêuticos não-convencionais em pacientes com doenças onco-hematológicas: um estudo de caso [trabalho de conclusão de curso]. Florianópolis (SC): Universidade Federal de Santa Catarina; 2006.

23. M ullany LC, Darmstadt GL, Khatry SK, Tielsch JM. Traditional massage of newborns in Nepal: implications for trials of improved practice. J Trop Pediatr 2005; 51(2):82-86.

24. Clement YN, M orton-Gittens J, Basdeo L, Blades A, Francis MJ, Gomes N, Janjua M, Singh A. Perceived efficacy of herbal remedies by users accessing primary healthcare in Trinidad. BM C Complement Altern M ed 2007. [periódico na Internet] 2007 [acessado $2007 \mathrm{dez}$ 5];7(4):[cerca de 9 p.]. Disponível em: http://www.biomedcentral.com/1472-6882/7/4

25. Tsao JCl, Zeltzer LK. Complementary and alternative medicine approaches for pediatric pain: a review of the state-of-the-science. Evid Based Complement Alternat M ed 2005; 2(2):149-159.

26. Niggemann B, Gruber C. Side-effects of complementary and alternative medicine. Allergy 2003; 58(8):707-716.

27. Ernst E. Serious adverse effects of unconvencional therapies for children and adolescents: a systematic review of recent evidence. Eur J Pediatr 2003; 162(2):72-80

28. Woolf AD. Herbal remedies and children: do they work? Are they harmful? Pediatrics 2003; 112:240-246.

29. Abebe W. Herbal medication: potential for adverse interactions with analgesic drugs. J Clin Pharm Ther 2002;27(6):391-401.

30. Jean $D, C y r C$. Use of complementary and alternative medicine in a general pediatric clinic. Pediatrics 2007; 120(1):e138-141.

Artigo apresentado em 13/07/2007

Aprovado em 14/12/2007 